\title{
Facile Preparation of Porous Carbon Derived from Industrial Biomass Waste as an Efficient $\mathrm{CO}_{2}$ Adsorbent
}

Caicheng Song, Wanyue Ye, Yingcen Liu, He Huang, Hao Zhang, Hua Lin, Rongwen $\mathrm{Lu}^{*}$ and Shufen Zhang

State Key Laboratory of Fine Chemicals, Dalian University of Technology, Dalian 116024, China

*Corresponding author.

E-mail address: lurw@dlut.edu.cn (Rongwen Lu) 


\section{Pictures}

The pictures of soybean cake involved in this work were taken in the oil mill which provided the soybean cake (Figure S1).
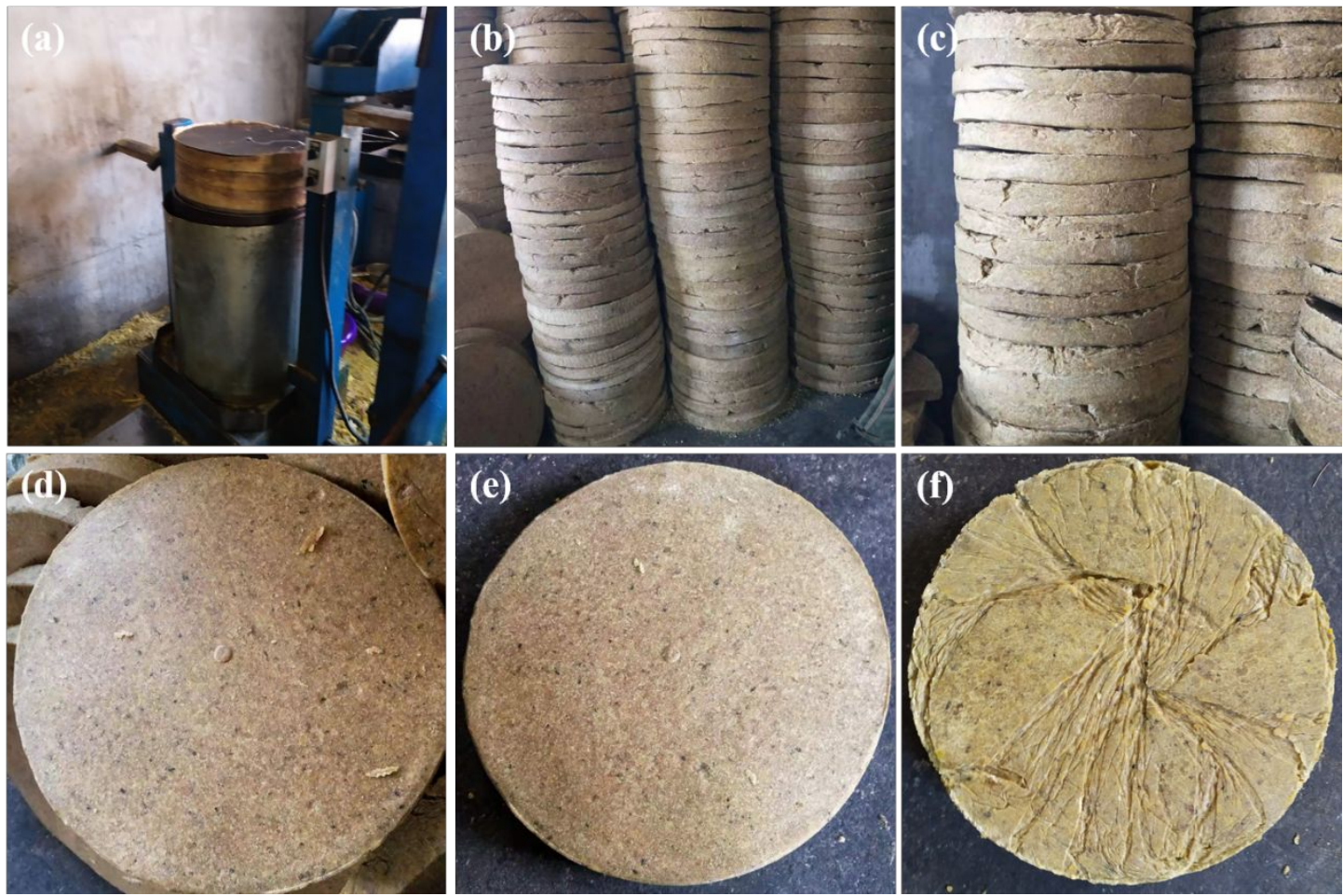

Figure S1. Pictures of the oil extraction equipment (a) and soybean cakes (b-f).

\section{Rising Program}

The carbonization temperature rising program is set according to the thermogravimetric analysis curve in Figure S2. The temperature is ramped up to $100{ }^{\circ} \mathrm{C}$ at $10{ }^{\circ} \mathrm{C} \mathrm{min}-1$ first, then $2{ }^{\circ} \mathrm{C} \min ^{-1}$ to $350{ }^{\circ} \mathrm{C}$ and keep the temperature for $90 \mathrm{~min}$, $5{ }^{\circ} \mathrm{C} \min ^{-1}$ to $600{ }^{\circ} \mathrm{C}$ and hold the temperature for another $90 \mathrm{~min}$. 


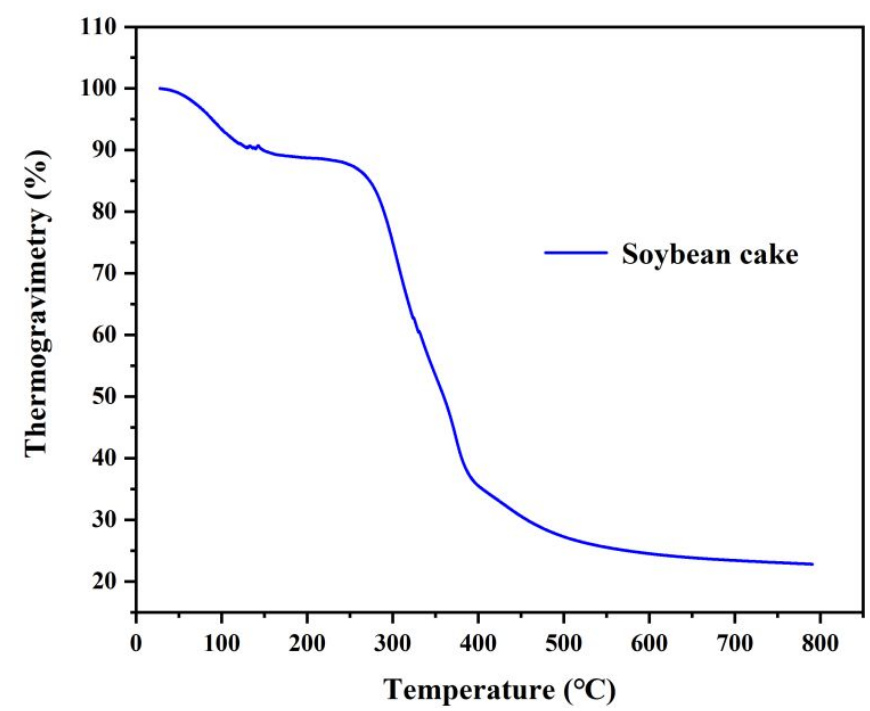

Figure S2. Thermogravimetric analysis of soybean cake.

\section{KOH Activation Mechanism}

The reaction equations involved in the activation process are as follows. ${ }^{1,2}$

$$
\begin{gathered}
6 \mathrm{KOH}+2 \mathrm{C} \rightarrow 2 \mathrm{~K}+3 \mathrm{H}_{2}+2 \mathrm{~K}_{2} \mathrm{CO}_{3} \\
\mathrm{~K}_{2} \mathrm{CO}_{3} \rightarrow \mathrm{K}_{2} \mathrm{O}+\mathrm{CO}_{2} \\
\mathrm{~K}_{2} \mathrm{CO}_{3}+\mathrm{C} \rightarrow \mathrm{K}_{2} \mathrm{O}+2 \mathrm{CO} \\
\mathrm{K}_{2} \mathrm{O}+\mathrm{C} \rightarrow 2 \mathrm{~K}+\mathrm{CO} \\
2 \mathrm{~K}+\mathrm{CO}_{2} \rightarrow \mathrm{K}_{2} \mathrm{O}+\mathrm{CO}
\end{gathered}
$$

To sum up, the high porosity of carbon materials is mainly attributed to chemical activation (redox reaction of the potassium compound with carbon), physical activation $\left(\mathrm{CO}, \mathrm{H}_{2}, \mathrm{CO}_{2}\right)$ and potassium metal to expand the carbon lattice.

\section{Prediction of Mixed Gas Selectivity by IAST}

Single-site Langmuir model (SSL) was used to fit the adsorption isotherms of single-component of $\mathrm{CO}_{2}$ or $\mathrm{N}_{2}$ at $0-1$ bar. ${ }^{3,4}$ 


$$
q^{e}=q_{1+K_{L} P_{e}}^{s}
$$

Where $\mathrm{K}_{\mathrm{L}}$ is the parameter of pure component Langmuir isotherm $\left(\right.$ bar $\left.^{-1}\right) . \mathrm{P}_{\mathrm{e}}$ is the pressure of bulk gas at equilibrium with the adsorbed phase $\mathrm{P}$ (bar). $\mathrm{q}_{\mathrm{s}}$ and $\mathrm{q}_{\mathrm{e}}$ are the saturation adsorbed amount and adsorbed amount of adsorbent, respectively.

The adsorption selectivity for mixed gas $\left(\mathrm{CO}_{2} / \mathrm{N}_{2}=15: 85\right)$ is predicted by the Eq.

$$
\mathrm{S}=\left(\frac{\mathrm{qCO}_{2}}{\mathbf{q}_{\mathrm{N}_{2}}}\right) /\left(\frac{\mathbf{P}_{\mathrm{CO}_{2}}}{\mathrm{P}_{\mathrm{N}_{2}}}\right)
$$

Where $\mathrm{S}$ is the selectivity for $\mathrm{CO}_{2} . \mathrm{P}$ is 0.15 and 0.85 , respectively. $\mathrm{q}$ is the adsorbed amount for $\mathrm{CO}_{2}$ or $\mathrm{N}_{2}$.

\section{The Isosteric Heats of Adsorption}

The isosteric heats of adsorption was calculated by the Clausius-Clapeyron equation. As follows:

$$
\mathbf{Q}_{\mathbf{s t}}=\mathbf{R T}^{2}\left(\frac{\partial \mathbf{l n p}}{\partial \mathbf{T}}\right)_{\mathbf{q}}
$$

$\mathrm{Q}_{\text {st }}$ is the isosteric heats of adsorption, $\mathrm{P}$ is the pressure, $\mathrm{R}$ is the gas constant, $\mathrm{T}$ is the temperature and $\mathrm{q}$ is the $\mathrm{CO}_{2}$ adsorption uptake.

\section{Multi-component Adsorption Breakthrough Curves}

Firstly, $0.69 \mathrm{~g}$ samples were purged for $2 \mathrm{~h}$ at $423 \mathrm{~K}$ under a He atmosphere. Then the temperature was cooled to $298 \mathrm{~K}$ for testing. $\mathrm{CO}_{2}$ and $\mathrm{N}_{2}$ entered the fixed bed reactor with a flow rate of 7.5 and $42.5 \mathrm{sccm}$, respectively. The total flow of multicomponent gas is $50 \mathrm{sccm}$. Finally, the concentration of each component of outlet gas changes with time was obtained (Breakthrough Curves). 
Scheme S1. Multi-Component Adsorption Breakthrough Curve Analyzer principle.

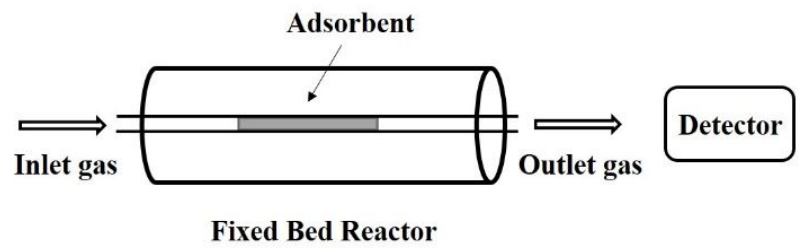

\section{Regeneration Cycle Test}

The samples were degassed for $6 \mathrm{~h}$ under vacuum at $423 \mathrm{~K}$ and cool naturally to room temperature before measurement. After the first adsorption test, the tested samples were degassed in $423 \mathrm{~K}$ for $3 \mathrm{~h}$ and then the second adsorption test was carried out. Five cyclic regeneration tests were performed by this way.

\section{Supplementary Figures and Data}

\section{Table S1. The Yield of All Samples}

\begin{tabular}{cccc}
\hline Samples & $\mathrm{m}_{1}^{\mathrm{a}}(\mathrm{g})$ & $\mathrm{m}_{2}^{\mathrm{a}}(\mathrm{g})$ & Yield $^{\mathrm{a}}(\%)$ \\
\hline CSC & 1.00 & 0.26 & 26 \\
CSC2-700 & 1.00 & 0.87 & 87 \\
CSC1-750 & 1.00 & 0.59 & 59 \\
CSC2-750 & 1.00 & 0.57 & 57 \\
CSC3-750 & 1.00 & 0.46 & 46 \\
CSC2-800 & 1.00 & 0.48 & 48 \\
\hline
\end{tabular}

aThe above data were taken from the average value of three tests.

$\mathrm{m}_{1}$ : mass of SCP and CSC.

$\mathrm{m}_{2}$ : mass of CSC and CSCX-Y.

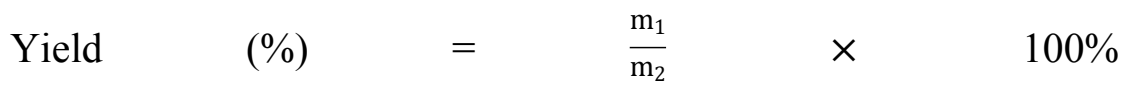



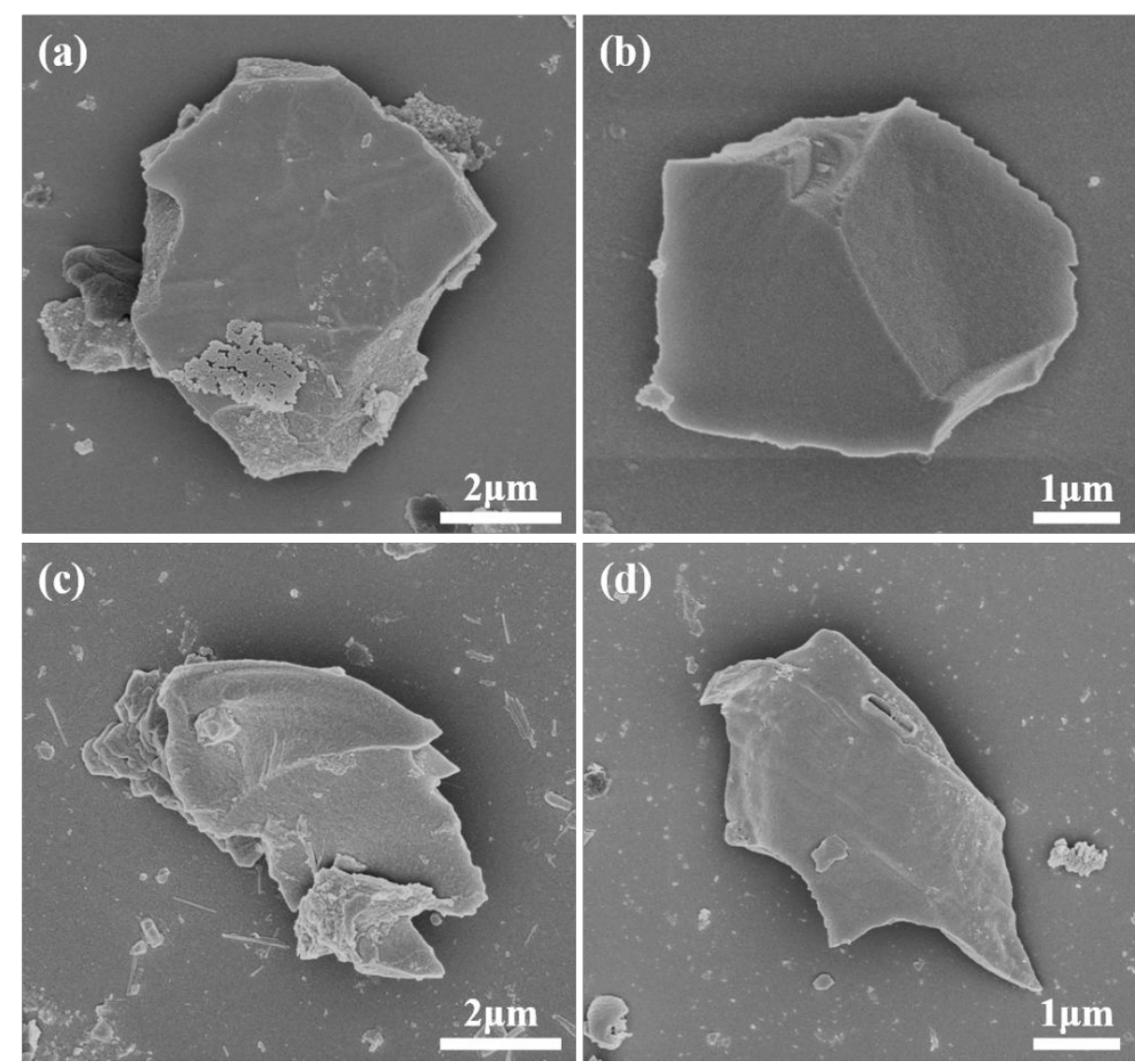

Figure S3. SEM images of (a) CSC2-700, (b) CSC1-750, (c) CSC3-750 and (d) CSC2800.

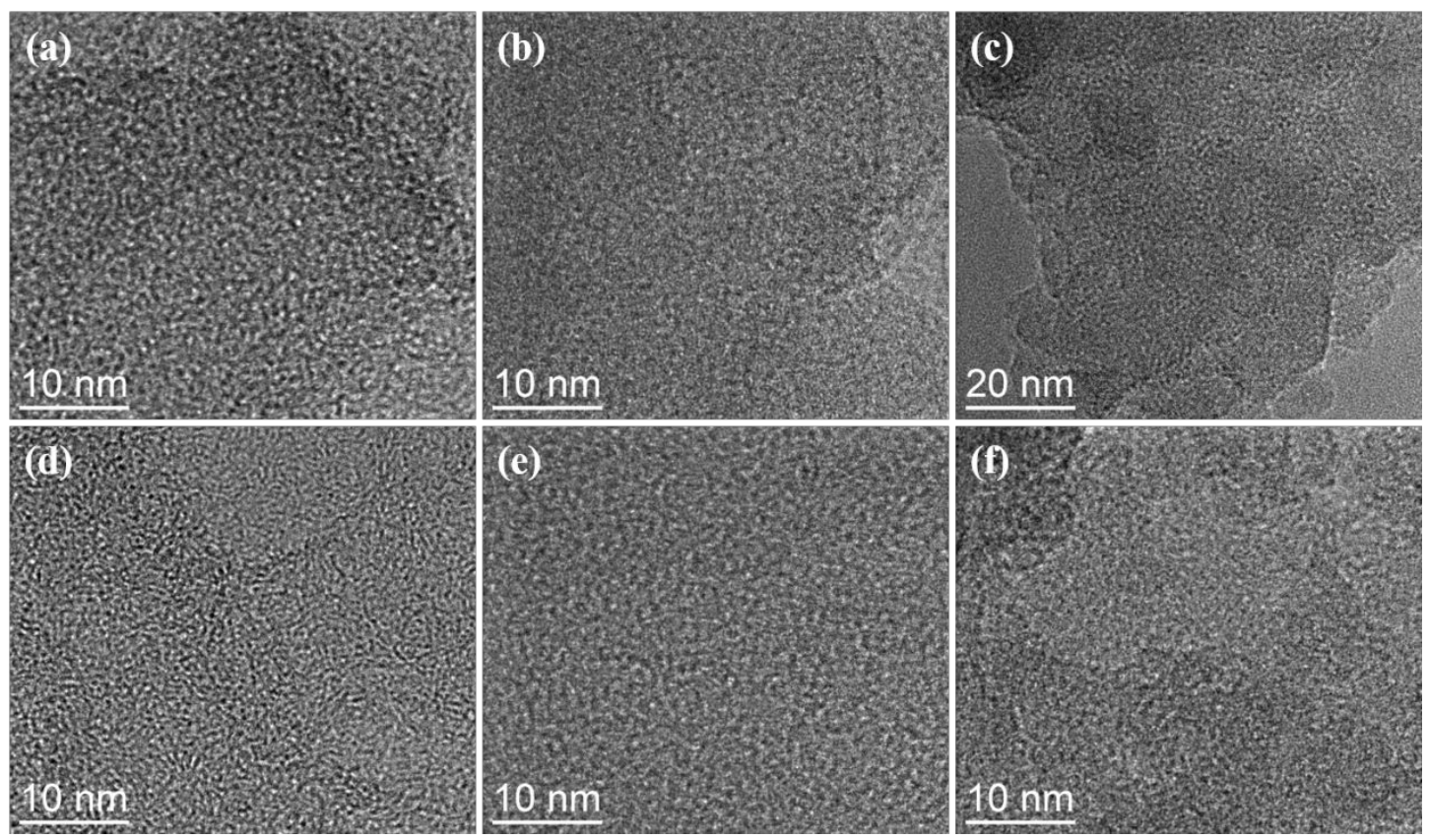

Figure S4. TEM images of (a) CSC2-700, (b) CSC1-750, (c, d) CSC2-750, (e) CSC3-

750 and (f) CSC2-800. 

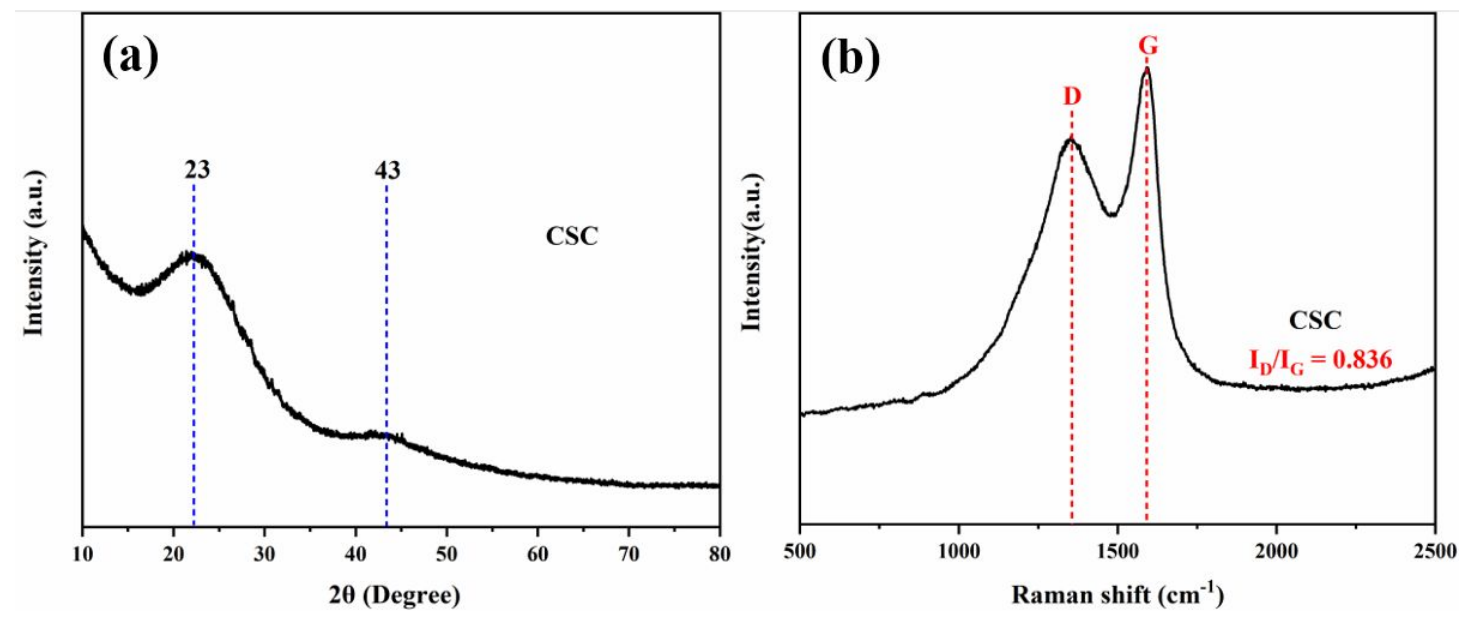

Figure S5. (a) XRD patterns and (b) Raman spectra of CSC.
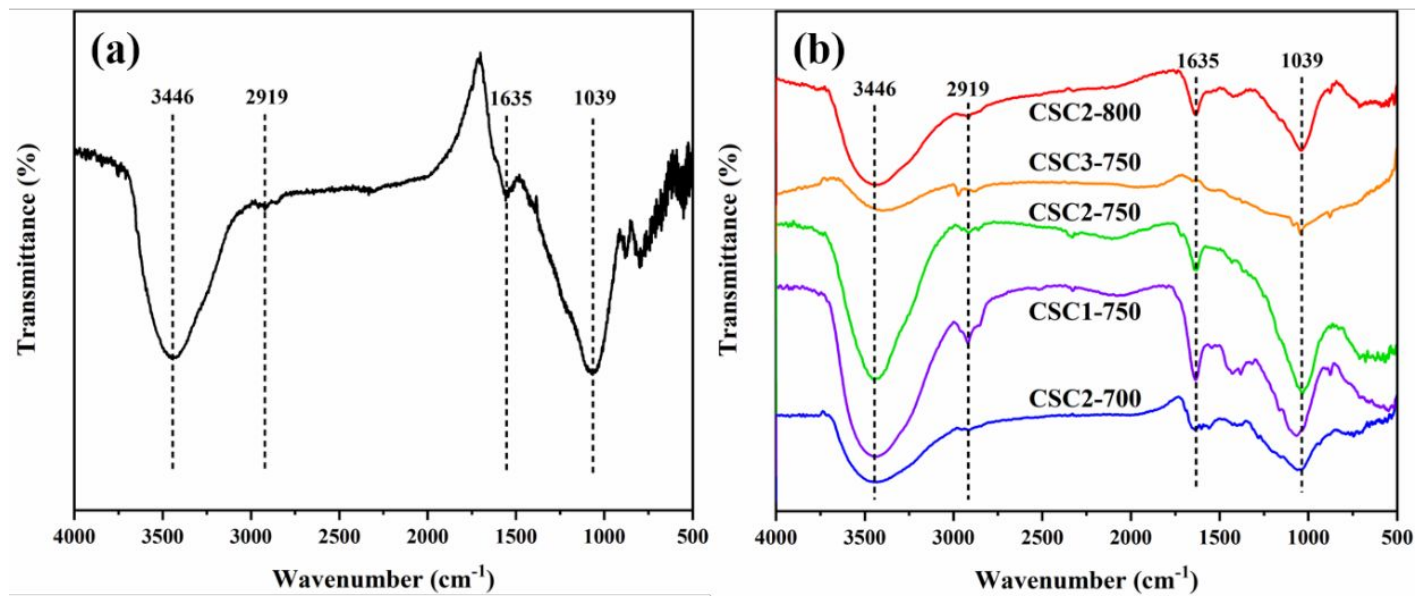

Figure S6. FT-IR spectra of (a) CSC and (b) CSCX-Y.

Table S2. Elemental Composition of Samples Derived from XPS Analysis

\begin{tabular}{cccc}
\hline \multirow{2}{*}{ Samples } & \multicolumn{3}{c}{ Element (atm\%) } \\
\cline { 2 - 4 } & $\mathrm{C}$ & $\mathrm{N}$ & $\mathrm{O}$ \\
\hline CSC & 85.44 & 2.82 & 11.73 \\
CSC2-700 & 80.68 & 0.95 & 18.37 \\
CSC1-750 & 52.51 & 1.19 & 46.30 \\
CSC2-750 & 72.28 & 1.02 & 26.70 \\
CSC3-750 & 72.09 & 0.63 & 27.29 \\
CSC2-800 & 68.22 & 0.94 & 30.84 \\
\hline
\end{tabular}



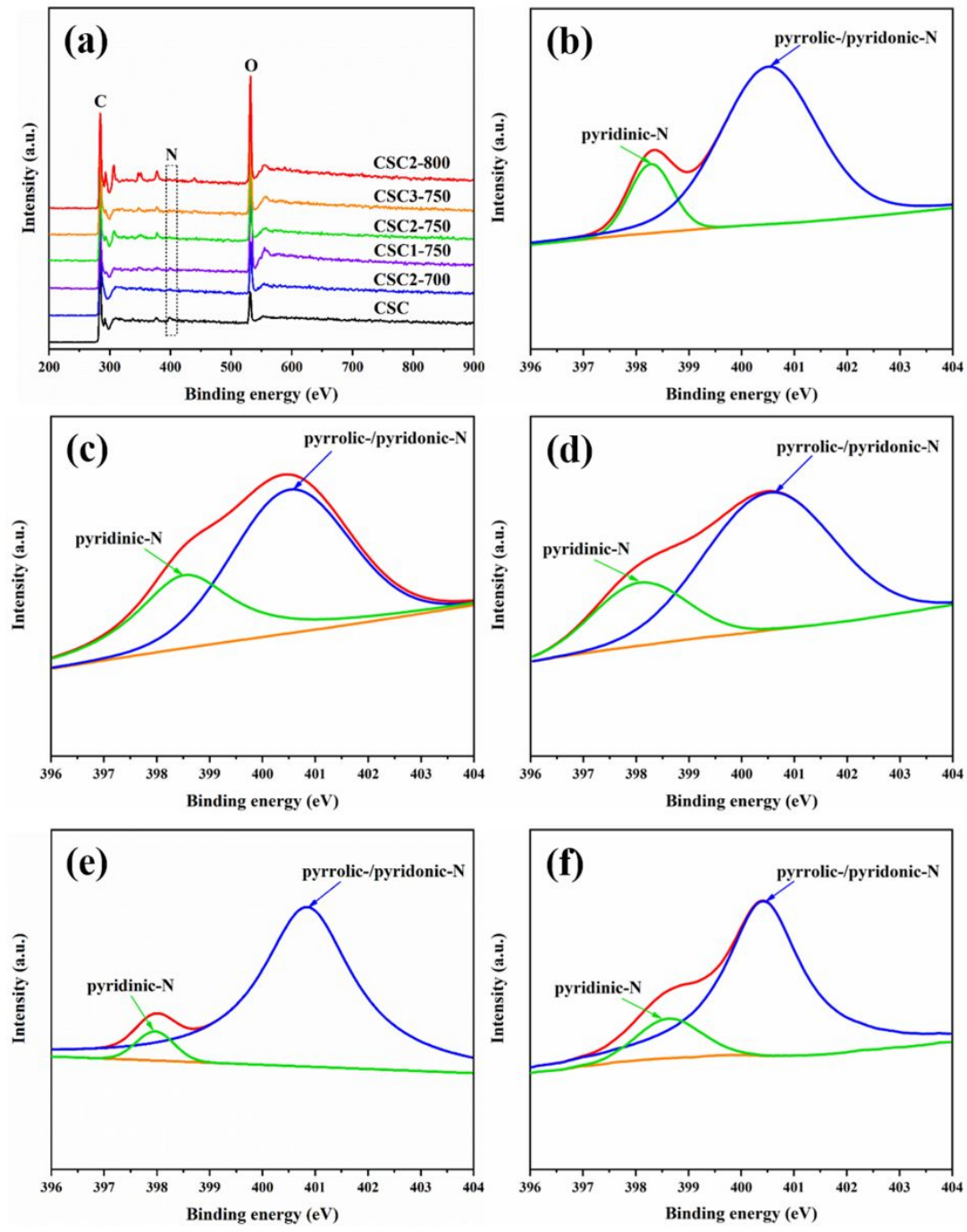

Figure S7. (a) XPS survey spectra of CSCX-Y; N 1s XPS spectra of (b) CSC2-700, (c) CSC1-750, (d) CSC2-750, (e) CSC3-750 and (f) CSC2-800.

Table S3. The Relative Content of Pyridinic-N and Pyrrolic-/ Pyridonic-N for All Samples

\begin{tabular}{ccc}
\hline \multirow{2}{*}{ Samples } & \multicolumn{2}{c}{ Relative Content (\%) } \\
\cline { 2 - 3 } & Pyridinic-N & Pyrrolic-/ Pyridonic-N \\
\hline CSC & 35 & 66 \\
CSC2-700 & 15 & 85 \\
CSC1-750 & 36 & 64 \\
CSC2-750 & 25 & 75 \\
CSC3-750 & 5 & 95 \\
CSC2-800 & 13 & 87 \\
\hline
\end{tabular}



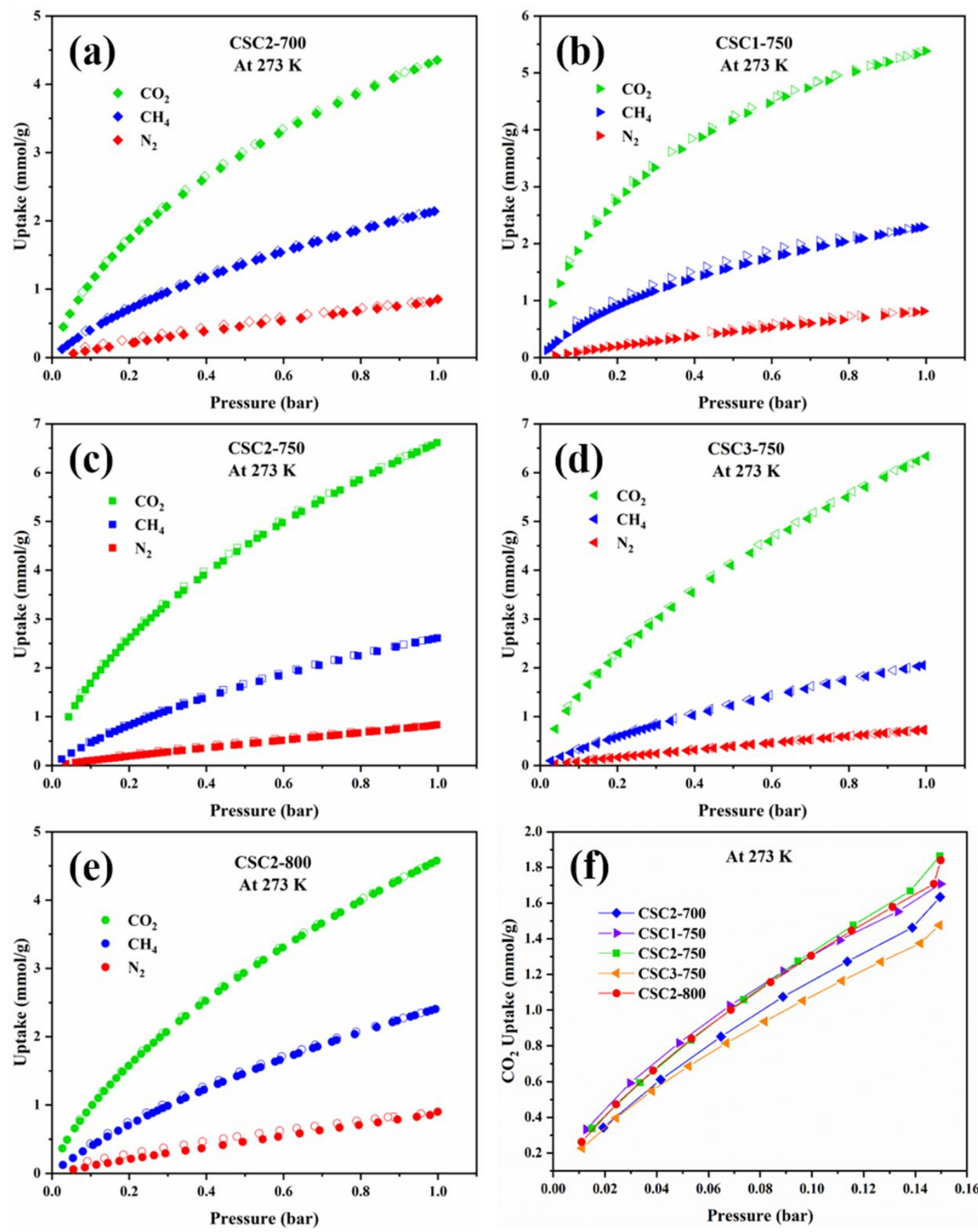

Figure S8. Gas adsorption isotherms of (a) CSC2-700, (b) CSC1-750, (c) CSC2-750,

(d) CSC3-750 and (e) CSC2-800 at $273 \mathrm{~K} ; \mathrm{CO}_{2}$ adsorption isotherms of (f) CSCX-Y at $273 \mathrm{~K}$. 

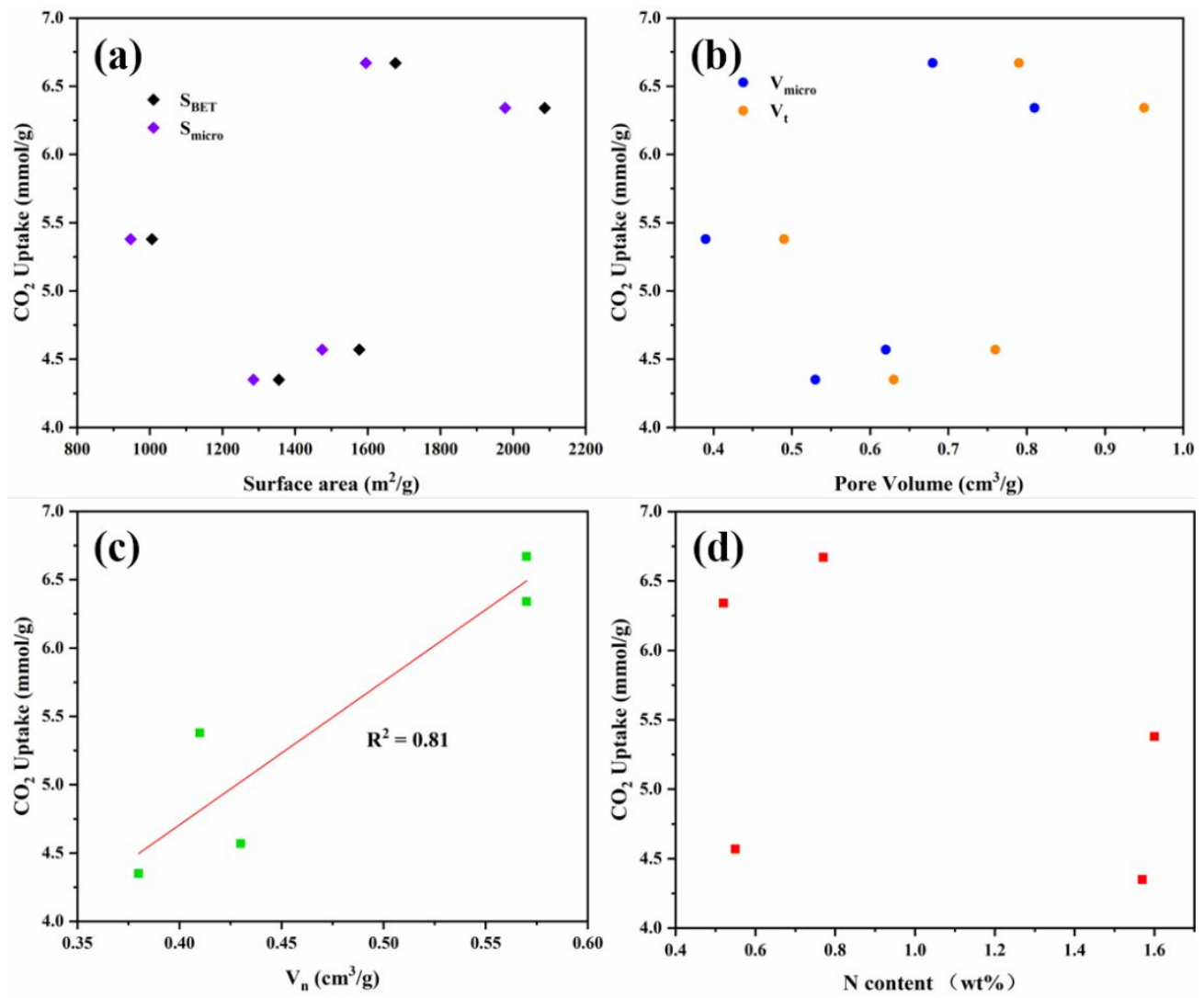

Figure S9. Relationship between $\mathrm{CO}_{2}$ adsorption capacity and (a) surface area, (b) pore volume, (c) $\mathrm{V}_{\mathrm{n}}$ and (d) $\mathrm{N}$ content at $273 \mathrm{~K}$ and 1 bar.

Table S4. $\mathrm{CO}_{2}$ Adsorption Uptake ${ }^{a}$

\begin{tabular}{ccc}
\hline \multirow{2}{*}{ Samples } & \multicolumn{2}{c}{$\mathrm{CO}_{2}$ uptake $(\mathrm{mmol} / \mathrm{g})$} \\
\cline { 2 - 3 } & $298 \mathrm{~K}$ & $273 \mathrm{~K}$ \\
\hline CSC2-700 & 0.74 & 1.63 \\
$\mathrm{CSC} 1-750$ & 1.26 & 1.71 \\
$\mathrm{CSC} 2-750$ & 1.26 & 1.86 \\
$\mathrm{CSC} 3-750$ & 1.01 & 1.48 \\
$\mathrm{CSC} 2-800$ & 0.96 & 1.84 \\
\hline
\end{tabular}

aMeasured at 0.15 bar. 

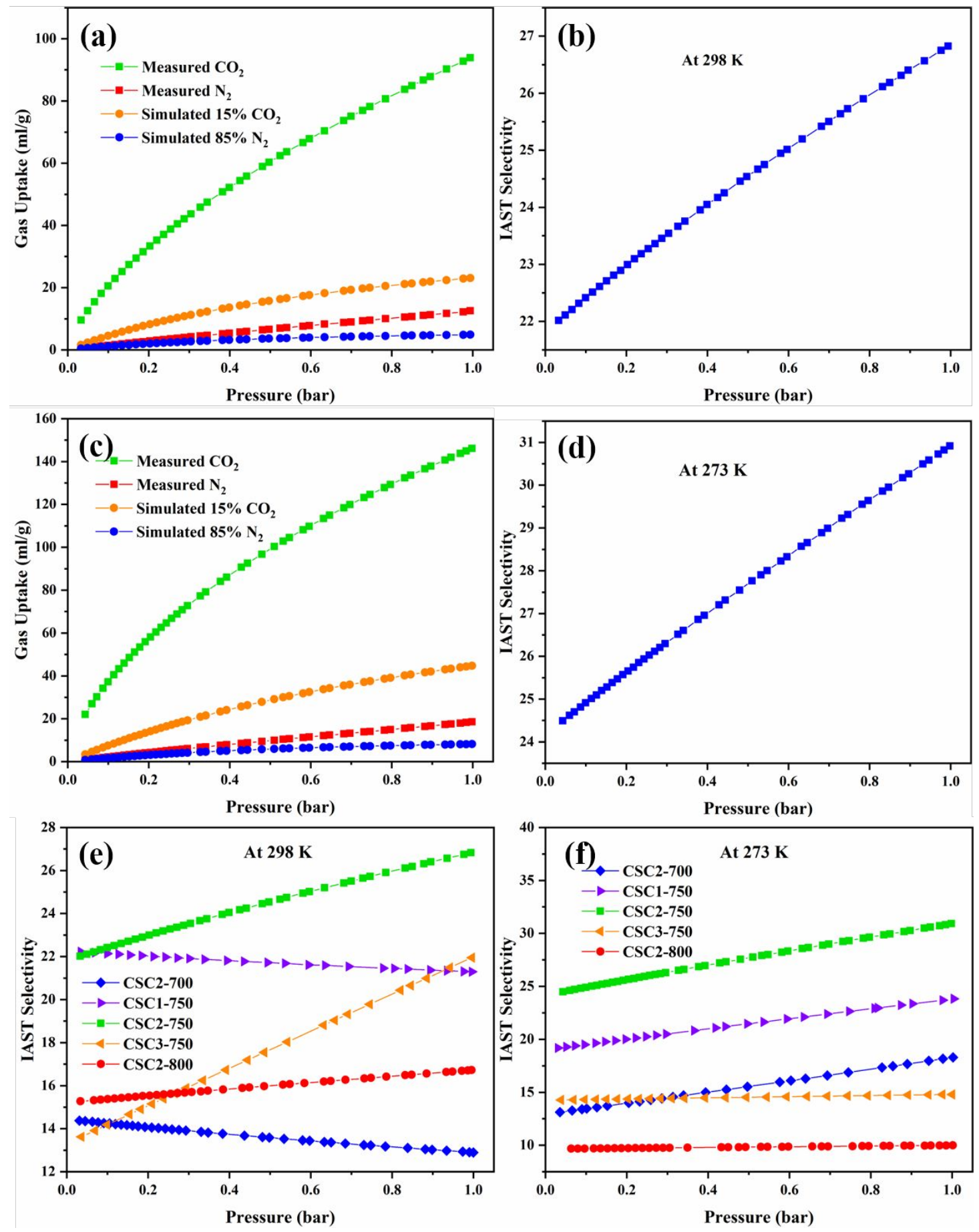

Figure S10. (a, c) the simulated gas adsorption isotherms $\left(15 \% \mathrm{CO}_{2}\right) ;(b, d)$ the IAST selectivity curves of CSC2-750 at 298 and $273 \mathrm{~K}$, respectively;(e, f) the IAST selectivity curves of CSCX-Y at 298 and $273 \mathrm{~K}$, respectively. 

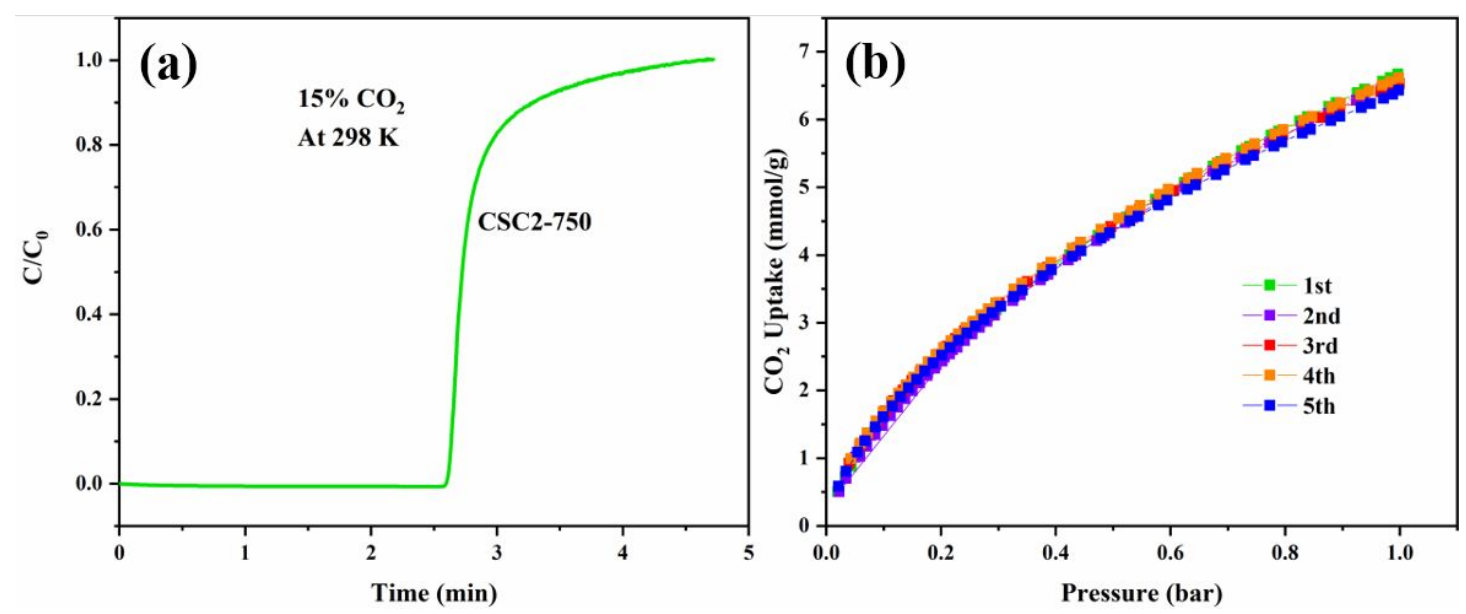

Figure S11. (a) Breakthrough curves $\left(15 \% \mathrm{CO}_{2}\right)$ and (b) a five-time cyclic regeneration tests of CSC2-750 at $273 \mathrm{~K}$.

Table S5. The Maximum Isosteric Heats of Adsorption of CSCX-Y

\begin{tabular}{ccc}
\hline \multirow{2}{*}{ Samples } & \multicolumn{2}{c}{ The maximum isosteric heats of adsorption $\left(\mathrm{Q}_{\mathrm{st}}, \mathrm{kJ} / \mathrm{mmol}\right)$} \\
\cline { 2 - 3 } & $\mathrm{CH}_{4}$ & $\mathrm{~N}_{2}$ \\
\hline CSC2-700 & 11 & 17 \\
CSC1-750 & 20 & 11 \\
CSC2-750 & 20 & 5 \\
CSC3-750 & 8 & 10 \\
CSC2-800 & 11 & 22 \\
\hline
\end{tabular}

Table S6. $\mathrm{CO}_{2}$ Adsorption Uptake of Different Adsorbents

\begin{tabular}{|c|c|c|c|c|c|}
\hline \multirow[b]{2}{*}{ Samples } & \multicolumn{3}{|c|}{$\mathrm{CO}_{2}$ uptake $(\mathrm{mmol} / \mathrm{g})$} & \multirow{2}{*}{$\begin{array}{l}\text { IAST } \\
\left(15 \% \mathrm{CO}_{2}\right)\end{array}$} & \multirow[b]{2}{*}{ Ref. } \\
\hline & $\begin{array}{l}298 \mathrm{~K} \\
(1 \mathrm{bar})\end{array}$ & $\begin{array}{l}273 \mathrm{~K} \\
(1 \mathrm{bar})\end{array}$ & $\begin{array}{c}298 \mathrm{~K} \\
\left(0.15 \mathrm{bar} / 15 \% \mathrm{CO}_{2}\right)\end{array}$ & & \\
\hline $\mathrm{a}-\mathrm{CL}$ & 4.26 & 6.04 & - & - & (5) \\
\hline PC1-780 & 3.87 & 6.02 & 1.28 & & (6) \\
\hline $\mathrm{H} 250-800$ & 3.71 & 5.23 & - & 11.2 & (7) \\
\hline CN-600-3 & 3.71 & 5.12 & $0.84\left(10 \% \mathrm{CO}_{2}\right)$ & 13 & (8) \\
\hline WSC-500-1 & 4.50 & 6.04 & - & $23\left(10 \% \mathrm{CO}_{2}\right)$ & (9) \\
\hline NGC-650-4 & 3.92 & 6.23 & $0.84\left(10 \% \mathrm{CO}_{2}\right)$ & 44 & $(10)$ \\
\hline NC-650-1 & 4.26 & 6.15 & - & 29 & (11) \\
\hline CRF-4 & 2.94 & 4.01 & 0.9 & - & (12) \\
\hline $\mathrm{PCN}-250\left(\mathrm{Fe}_{2} \mathrm{Co}\right)$ & 3.87 & 7.33 & - & 47 & (13) \\
\hline caCTF1-70 & 3.55 & 6.00 & $1.45(273 \mathrm{~K})$ & & (14) \\
\hline ACSD-2700 & 4.90 & - & 1.1 & & $(15)$ \\
\hline SC-TPB F & 3.00 & - & 0.65 & 26 & (16) \\
\hline CSC2-750 & 4.19 & 6.61 & $1.26 / 1.28$ & 27 & This work \\
\hline
\end{tabular}




\section{REFERENCES}

(1) Rao, L.; Ma, R.; Liu, S.; Wang, L.; Wu, Z.; Yang, J.; Hu, X. Nitrogen enriched porous carbons from D-glucose with excellent $\mathrm{CO}_{2}$ capture performance. Chem. Eng. J. 2019, 362, 794-801.

(2) Li, J.; Michalkiewicz, B.; Min, J.; Ma, C.; Chen, X.; Gong, J.; Mijowska, E.; Tang, T. Selective preparation of biomass-derived porous carbon with controllable pore sizes toward highly efficient $\mathrm{CO}_{2}$ capture. Chem. Eng. J. 2019, 360, 250-259.

(3) Myers, A. L.; Prausnitz, J. M. Thermodynamics of mixed-gas adsorption. AIChE J. 1965, 11 (1), 121 127.

(4) Zhao, Y.; Liu, X.; Yao, K. X.; Zhao, L.; Han, Y. Superior Capture of $\mathrm{CO}_{2}$ Achieved by Introducing Extra-framework Cations into N-doped Microporous Carbon. Chem. Mater. 2012, 24 (24), 4725-4734.

(5) Wang, R.; Wang, P.; Yan, X.; Lang, J.; Peng, C.; Xue, Q. Promising porous carbon derived from celtuce leaves with outstanding supercapacitance and $\mathrm{CO}_{2}$ capture performance. ACS Appl. Mater. Interfaces 2012, 4 (11), 5800-6.

(6) Li, D.; Ma, T.; Zhang, R.; Tian, Y.; Qiao, Y. Preparation of porous carbons with high low-pressure $\mathrm{CO}_{2}$ uptake by $\mathrm{KOH}$ activation of rice husk char. Fuel 2015, 139, 68-70.

(7) Parshetti, G. K.; Chowdhury, S.; Balasubramanian, R. Biomass derived low-cost microporous adsorbents for efficient $\mathrm{CO}_{2}$ capture. Fuel 2015, 148, 246-254.

(8) Yue, L.; Xia, Q.; Wang, L.; Wang, L.; DaCosta, H.; Yang, J.; Hu, X. $\mathrm{CO}_{2}$ adsorption at nitrogendoped carbons prepared by $\mathrm{K}_{2} \mathrm{CO}_{3}$ activation of urea-modified coconut shell. J. Colloid Interface Sci. 2018, 511, 259-267.

(9) Rao, L.; Liu, S.; Wang, L.; Ma, C.; Wu, J.; An, L.; Hu, X. N-doped porous carbons from lowtemperature and single-step sodium amide activation of carbonized water chestnut shell with excellent $\mathrm{CO}_{2}$ capture performance. Chem. Eng. J. 2019, 359, 428-435.

(10) Yue, L.; Rao, L.; Wang, L.; An, L.; Hou, C.; Ma, C.; DaCosta, H.; Hu, X. Efficient $\mathrm{CO}_{2}$ adsorption on nitrogen-doped porous carbons derived from D-glucose. Energy Fuels 2018, 32 (6), 6955-6963.

(11) Yang, M.; Guo, L.; Hu, G.; Hu, X.; Xu, L.; Chen, J.; Dai, W.; Fan, M. Highly cost-effective nitrogendoped porous coconut shell-based $\mathrm{CO}_{2}$ sorbent synthesized by combining ammoxidation with $\mathrm{KOH}$ activation. Environ. Sci. Technol. 2015, 49 (11), 7063-70.

(12) Du, J.; Li, W.-C.; Ren, Z.-X.; Guo, L.-P.; Lu, A.-H. Synthesis of mechanically robust porous carbon monoliths for $\mathrm{CO}_{2}$ adsorption and separation. J. Energy Chem. 2020, 42, 56-61.

(13) Chen, Y.; Qiao, Z.; Huang, J.; Wu, H.; Xiao, J.; Xia, Q.; Xi, H.; Hu, J.; Zhou, J.; Li, Z. Unusual moisture-enhanced $\mathrm{CO}_{2}$ capture within microporous PCN-250 frameworks. ACS Appl. Mater. Interfaces 2018, 10 (44), 38638-38647.

(14) Lee, Y. J.; Talapaneni, S. N.; Coskun, A. Chemically activated covalent triazine frameworks with enhanced textural properties for high capacity gas storage. ACS Appl. Mater. Interfaces 2017, 9 (36), 30679-30685.

(15) Hirst, E. A.; Taylor, A.; Mokaya, R. A simple flash carbonization route for conversion of biomass to porous carbons with high $\mathrm{CO}_{2}$ storage capacity. J. Mater. Chem. A 2018, 6 (26), 12393-12403.

(16) Alahmed, A. H.; Briggs, M. E.; Cooper, A. I.; Adams, D. J. Post-synthetic fluorination of Schollcoupled microporous polymers for increased $\mathrm{CO}_{2}$ uptake and selectivity. J. Mater. Chem. A 2019, 7 (2), 549-557. 